\title{
The Effect of Context on the EFL Learners' Idiom Processing Strategies
}

\author{
Gholamreza Rohani ${ }^{1}$, Saeed Ketabi ${ }^{1} \&$ Mansoor Tavakoli ${ }^{1}$ \\ ${ }^{1}$ English Department, Faculty of Foreign Languages, University of Isfahan, Isfahan, Iran \\ Correspondence: Gholamreza Rohani, English Department, Faculty of Foreign Languages, University of Isfahan, \\ Isfahan, Iran. E-mail: rrohani@gmail.com
}

Received: May 21, $2012 \quad$ Accepted: June 14, $2012 \quad$ Online Published: July 27, 2012
doi:10.5539/elt.v5n9p104 URL: http://dx.doi.org/10.5539/elt.v5n9p104

\begin{abstract}
The present study investigated the effect of context on the strategies the EFL learners utilized to process idioms. To do so, ten Iranian intermediate EFL learners were randomly assigned to two groups who then attended a think-aloud session. The 5 subjects in the first group were exposed to an animated cartoon including 23 unfamiliar idioms while their counterparts in the second group were exposed to the written version of the same material. The subjects of the two groups were asked to verbally report their thought processes when trying to define the unfamiliar idioms. The data thus gathered form the small sample revealed 8 major strategies which were then used to prepare a questionnaire to be administered to a larger sample. The new sample included 60 subjects randomly assigned to two groups of 30.The first group as in the think-aloud session was exposed to the animated cartoon while the other read the respective script. The subjects in both groups were asked to check the strategies they resorted to when guessing the meaning of unfamiliar idioms. The results of the study showed both inter and intra-group differences confirming the effect of context on the strategies applied in processing unfamiliar idioms.
\end{abstract}

Keywords: idioms, video graphic context, written context, idiom processing strategies

\section{Introduction}

An Idiom in a simple definition is a multi-word expression whose components are fixed or semi-fixed and whose meaning is different form the sum of its parts.Idiomatic expressions are figurative expressions such as "as cool as cucumber" or "beat around the bush" which as suggested by some are regarded as single lexical units. They are stored and retrieved similar to other words in the mental lexicon (Qualls, O'Brien, Blood, \& Hammer, 2003).

Ellis (1997) suggests that sufficient knowledge and appropriate use of idioms in a second language is an important indicator of the language learners' communicative competence. According to Liu (2008), many L2 learners, especially intermediate and advanced students, are eager to learn more idioms due to the fact that these learners have had a good exposure to the target language and hence have learnt to appreciate the value and the importance of idioms, including their vividness and effectiveness in communicative tasks. Gibbs (1999) states that idioms are one of the commonest forms of figurative language which differ in many aspects such as transparency, compositionality and frozenness. In other words, Idioms are multi-faceted expressions whose proper definition involves various viewpoints and approaches and that's why despite various attempts by researchers (e.g., Barkema, 1996; Cowie, 1998; Glaser, 1998) they aren't still well-defined. However, the discrepancy between what is said and what is meant remains the essential characteristic of idiomatic expressions which in turn makes their verbatim processing almost impossible.

\section{Review of the Literature}

\subsection{Theoretical Background}

Different hypotheses have been offered in the literature to explain the way idioms are processed. Idiom-list model by Bobrowand Bell (1973) states that when encountering an idiom, we first interpret it literally. If a literal meaning does not fit the context in which the expression is used, we search for that idiom in our mental idiom lexicon and then chooseits figurative meaning. The second model for idiom processing is the lexical representation model (Swinney \& Cutler, 1979). It depicts idioms as long words retrieved form the mental lexicon along with all other words. In a simultaneous processing of both literal and figurative meanings, it's the context which determines the winner. The third model, the direct access model (Gibbs, 1980; Schweigert, 1986), 
is not far from the lexical representation model. Accordingly, we usually disregard the literal meaning of an idiomatic expression and the figurative meaning is directly derived from the mental lexicon.Glucksberg (1993) somehow confirms this model by stating that idiom access is normally completed more quickly because it does not require the lexical, syntactic, and semantic processing needed for full linguistic analysis. The next hypothesis, the Configuration Model (Cacciari \& Tabossi, 1988), states that the literal meaning of the words comprising an idiom is activated and remains so during its processing. Still the other recent model, idiom decomposition hypothesis, developed by Gibs and his colleagues (Gibbs \& Nayak, 1989; Gibbs, Nayak \& Cutting, 1989) emphasizes the feature of compositionality. An idiom is decomposable when its individual components contribute to its figurative meaning. Idioms whose individual components do not make such a contribution are non-decomposable. The words comprising a rather decomposable idiom are retrieved from the learner's mental lexicon and then combined with the other components while the meaning of a non-decomposable idiom is retrieved directly from the lexicon. The last major idiom comprehension model is the hybrid model (Titone \& Connine, 1999). Based on an eye-tracking study they concluded that both the literal and figurative meanings of the idioms are automatically activated.But for non-decomposable idioms it takes more time to come to a contextually appropriate meaning.

While most of the models proposed for idiom comprehension are based on L1 studies, research on processing of L2 idioms and figurative language has mainly focused on L1 transfer (Irujo, 1986; Kellerman, 1983).

In general, studies on idiom comprehension focus on three major factors, namely, semantic transparency, familiarity, and context. According to Nippold and Taylor (1995) Transparency is the degree of agreement between the literal and figurative meanings of an idiom. The meaning of a transparent idiom matches well with the image it depicts. An idiom like "go by the book" is highly transparent because its literal meaning, to follow directions in a book exactly, is closely associated with its nonliteral meaning, to closely follow rules and regulations. But the expression "beat around the bush" is not transparent, because its literal meaning is not associated with its nonliteral meaning, reluctance to talk about a topic. Semantic transparency is not a fixed and absolute feature. It can be regarded on a continuum from highly literal to highly figurative correspondence. Many idioms are neither completely transparent nor opaque. The concept of transparency can also be discussed in terms of decomposition (Glucksberg, 2001). The concept of Compositionality states that idioms are at least partly decomposable, and speakers can intuitively understand the way the components of the idiom contribute to its whole meaning and this notion can determine the syntactic behavior of the idioms: the more an idiom seems analyzable and hence transparent, the more likely that idiom will be treated as syntactically flexible (Gibbs \& Nayak, 1989). Nunberg, Sag and Wasow (1994) suggest thatidiomatic phrases are, for the large part, semantically compositional, and seldom totally rigid. They believe that the words in idioms can appear in more than one form though not in all forms. They don't reject that some idioms are inflexible, but claim that such idioms comprise just a small part.Though the claims of the idiom decomposition are now widely accepted (Glucksberg, 2001), the empirical evidence to support the view is controversial. Gibbs et al. (1989) found that decomposable idioms are more flexible and are processed faster than non-decomposable and rigid ones, while Swinney and Cutler (1979) didn't find any time difference in the processing of flexible and nonflexible idioms. In another study Titone and Connine (1999) found that non-decomposable idioms were read more slowly when the sentential context preceded the idiom than when it followed it.

Familiarity, the other main factor influencing idiom comprehension, is the frequency of occurrence of an idiom in the language. While an idiom such as "call it a day" is frequently used in English, another idiom like"get your wires crossed" is one that is rarely used. The data from Nippold and Taylor (1995) showed that high-familiarity idioms were easier to understand than those of low familiarity. The findings are in agreement with the "language experience" hypothesis, the view that frequency of exposure enhances learning the meanings of idioms.

The third major factor influencing idiom comprehension is context. Idiomatic expressions are understood in relation to the context in which they are used. As a result, the skills used to process and understand language in context are thought to be important for the development of idiom understanding (Levorato \& Cacciari, 1995).

Many studies are done to enhance the process of learning idioms with regard to the 3 major factors mentioned above. The results of an experiment by Boers, Lindstromberg, Littlemore, Stengers and Eyckmans (2008) suggest that creating a connection between figurative idioms and their literal origins can indeed be a pedagogically effective technique to help learners remember the given expressions.One way to help learners to create such connections is the use of images. This is also in agreement with the dual coding theory (Paivio, 1986) according to which, the association of verbal information with a mental image is beneficial as it creates an additional pathway for the recollection of theverbal information. Boers, Eyckmans and Stengers (2006) based on the results of their study state that individuals whose cognitive style shows a predisposition for thinking in mental pictures generally obtain better scores in the meaning multiple-choice and the gap-fill exercises compared 
with their low-imager peers. In their study Boers et al. compared agroup of students exposed to on-line exercises in which the explanation about the originof the idioms was accompanied by a picture (a photograph or a drawing) with anothergroup who was presented with no pictures to elucidate the verbal input. The performance under the picture-enhanced version revealed a significant $(p<.02)$ improvement in themeaning multiple-choice exercises.

\subsection{Verbal Protocols}

One of the common ways to investigate the processes involved in comprehension is verbal reports or verbal protocols. As Kasper (1998) describes, Verbal reports are oral records of thoughts, provided by subjects during or immediately after completing a task. Research on the reliability of such methods of verbal reporting has shown that think aloud (during the task) procedures provide more reliable information and less task interference than introspection does (Ericsson \& Simon, 1993).

Cooper (1999) employed think-aloud to understand how adult second language learners processed idioms since this methodology allowed for the evaluation of the usually silent processes involved in reading comprehension. To give the idiomatic expressions context, he included more literal idioms (e.g. to see eye to eye) and more oral idioms or slang (e.g. what's cooking?). All were embedded in one to two sentences. Some of the limitations of his study were lack of controlling factors such as familiarity and context. The sample was also linguistically variable in their first languages.

\section{Significance of the Study}

Owing to the important role assigned to idioms in L2 acquisition and the difficulties EFL learners experience in their learning, finding a way to enhance their learning is of high importance. But this would be possible if there is an awareness of the processes involved in comprehending idioms. Such awareness can help the language teaching practitioners and material developers in decision making which would in turn lead to the development of a better curriculum.

Previous developmental research on idiom processing has mainly focused on L1 speakers. Furthermore, those few studies focusing on L2 learners have mainly paid attention to L1 transfer and have hardly attempted to investigate and compare the processes involved in comprehending idioms in different contexts. The present study is an attempt to fill such a gap.

\section{Research Questions}

The present study tries to investigate the major processes involved in idiom comprehension by Iranian intermediate EFL learners in the traditional written context and an animated cartoon context. The study tries to answer the following questions:

1. What are the major strategies involved in idiom processing while the L2 learners are exposed to a written context?

2. What are the major strategies involved in idiom processing while the L2 learners are exposed to a video-graphic context?

3. How far do the strategies followed by EFL learners to process unfamiliar idioms in written and video-graphic contexts differ?

\section{Methodology}

\subsection{Participants}

The participants of the study were 70 intermediate EFL learners (16 males and 54females) studying English language at Shahid Bahonar University of Kerman. They were chosen out of 104 students in three different classesbased on their scores on the Oxford Placement Test .The subjects were then assigned to 4 groups. 10 subjects in the same class made up the first two groups for the think aloud process. The remaining 60 subjects were also divided into 2 groups of 30 to answer the questionnaires. The subjects were majoring in either English translation or English literature, and their average age was 20. All had studied English in Iran for an average of 8 years.

\subsection{Materials}

The subjects were exposed to the selected idioms through the "Symphony in Slang", an animated cartoon by Tex Avery, and its script. The cartoon animation included 27 idioms of daily use based on Oxford Advanced Learners' Dictionary (2008 edition) and Collins COBUILD Dictionary of Idioms (2002 edition).

An idiom familiarity judgment task following Nippold and Rudzinski (1993) was the instrument used to choose the idioms rather unfamiliar to the subjects. They were asked to choose their familiarity level, the frequency of previously encountering that specific idiom in spoken or written contexts, in a 4 point scale $(1=$ many times, $2=$ 
a few times, 3 = once and $4=$ never).

Think-aloud was the instrument used to determine the most common strategies the language learners applied to comprehend unfamiliar idioms in the two different contexts.

A questionnaire constructed based on the results of the think-aloud sessions was the next instrument used to determine the specific strategyused by subjects in large samplesto define each of the unfamiliar idioms.

\subsection{Procedures}

To identify the major strategies that Iranian intermediate EFL learners refer to while guessing the meaning of unfamiliar idioms, first of all, a familiarity judgment task was administered to all 70 subjects to select the rather unfamiliar idioms. Each of the subjects was given a booklet including 27 more frequently used idioms form "Symphony in slang" by Tex Avery consulting Oxford Advanced Learners' Dictionary (2008 edition) and Collins COBUILD Dictionary of Idioms (2002 edition). The subjects were asked to assess the familiarity level of each idiom by indicating how frequently they had encountered it before in written or spoken forms, using a 4-point scale $(1=$ many times; $2=$ a few times; $3=$ once and $4=$ never $)$. The highest score for each idiom in this task was 4 with a lower score indicating more familiarity.Just the idioms with the average scores of 3 to 4 were selected resulting in a list including 23 items. The idioms "raining cats and dogs", "eat like a horse", "had his hands full" and "died laughing" were rejected at this stage due to their being more familiar to the subjects.

In the next stage of the study, 10 subjects all in the same class attended a session to get familiar with verbal reports. The researcher started with verbally reporting his thought processes while doing a multiplication task. Two of the subjects were asked to practice it and then for further familiarity the researcher gave them a handout including a passage with 3 idioms. The researcher then read the passage and verbally reported the processes he followed to guess the meaning of one of those idioms. Two volunteers went on with the rest of the job.The 10 subjects were then randomly assigned to 2 groups of 5 hereafter referred to as animation and text groups to attend a think-aloud session. The subjects of the animation group entered the researcher's office individually and were seated at a desk with a laptop and high quality speakers attached to before them. To help the subjects to further focus on their cognitive processes, the researcher sat in the corner of the room in a way not to face the subjects directly. After a short explanation about the story, they were exposed to the animated cartoon and were asked to orally report their thought processes involved in defining each of the 23 paused at idioms.

The subjects in the text group also entered the office individually who were then given the transcript of the cartoon animation with the same 23 unfamiliar idioms underlined. After the researcher's brief explanation about the story, they were asked to read the text and verbally report their thought processes when trying to define the underlined idioms. As seen in both cases there was no teaching session, and the subjects of the two groups were only once exposed to their relevant materials.

The think-aloud sessions with both groups were wholly audio-taped and the protocols were transcribed and then analyzed based on the research objectives. To make sure about the reliability of the coding process, the researcher recoded the data after a week rendering almost similar results.As shown in table, the results of the think-aloud process indicated 11 strategies the subjects referred to when guessing the meaning of unfamiliar idioms. The coding process was mainly based on the existing literature though the category "Focusing on the accompanying animated picture" was new.

Table 1. Strategies used by subjects in animation and text groups

\begin{tabular}{ll}
\hline Major categories & Subcategories \\
\hline Idiom based & $\begin{array}{l}\text { Focusing on a key word in idiom } \\
\text { Focusing on the literal meaning } \\
\text { Reference to the origin of the idiom } \\
\text { paraphrasing and elaboration of the idiom }\end{array}$ \\
\hline Respondent based & $\begin{array}{l}\text { Drawing on background knowledge } \\
\text { Visualization (forming a mental image of the written idiom) } \\
\text { Requesting information about the idiom in general or unfamiliar words }\end{array}$ \\
\hline L1 based & Translating the idiom into first language or Finding an equivalent in Persian \\
\hline Context based & drawing on the accompanying animated picture \\
& Drawing on the written context (sentence, paragraph or the whole text) \\
\hline others & Resorting to wild guesses \\
\hline
\end{tabular}


8 single strategies out of the above 11 were selected as the major strategies referred to by the subjects of both groups, which were then used to prepare a questionnaire to be administered to a larger sample including 60 subjects (2 groups of 30 ). This was done to get more accurate results particularly while just single (not combined) strategies were the focus of attention and also for the purpose of triangulation. 3 strategies, i.e. referring to the origin of the idiom, wild guessing and requesting information, which had the lowest frequencies in the think-aloud experiment, were excluded in the questionnaire due to their low statistical value and the size of the sample.

The questionnaire (see appendix A) thus consisted of 23 rather unfamiliar idioms with 8 columns for the subjects to check the single strategy they used in defining them plus two other choices "combination of strategies "and "other strategies".

Following the think-aloud sessions, the subjects of the two groups were exposed to the script and animated cartoon of "symphony in slang "respectively. They were then asked to check the main strategy they used to defineeachunderlined or paused at idiom. In case they resorted to more than one strategy, they checked the column "combined". The column "other" was checked when the strategy the subjects used was not among the existing categories.

\section{Results}

The strategies used by the subjects attending the think-aloud session were examined for shared features and then classified mainly based on the existing literature.

Table 2 shows the frequency of different strategies that each subject resorted to in order to define the 23 idioms. Occasionally, the subjects resorted to more than one strategy to define a single idiom.

Table 2. The frequencies of different strategies in the think-aloud session

\begin{tabular}{|c|c|c|c|c|c|c|c|c|c|c|c|}
\hline \multicolumn{12}{|l|}{ Group1 (Text) } \\
\hline $\begin{array}{ll}\text { Strategies } \\
\text { subjects }\end{array}$ & S1 & S2 & S 3 & S4 & S5 & S6 & S7 & $\mathrm{S} 8$ & S9 & $\mathrm{S} 10$ & S11 \\
\hline 1 & 4 & 6 & 2 & 2 & 1 & 1 & 1 & 4 & 4 & 2 & 0 \\
\hline 2 & 3 & 4 & 2 & 2 & 2 & 0 & 1 & 6 & 4 & 1 & 0 \\
\hline 3 & 7 & 6 & 4 & 3 & 1 & 0 & 2 & 3 & 3 & 4 & 0 \\
\hline 4 & 3 & 4 & 2 & 1 & 0 & 0 & 1 & 2 & 3 & 2 & 0 \\
\hline 5 & 5 & 4 & 4 & 2 & 0 & 2 & 2 & 4 & 6 & 5 & 0 \\
\hline $\begin{array}{l}\text { Total } \\
\text { frequency }\end{array}$ & 22 & 24 & 14 & 10 & 4 & 3 & 7 & 19 & 20 & 14 & 0 \\
\hline \multicolumn{12}{|l|}{ Group2 (Animation) } \\
\hline 1 & 4 & 5 & 1 & 0 & 1 & 0 & 1 & 2 & 4 & 4 & 4 \\
\hline 2 & 5 & 2 & 3 & 0 & 1 & 1 & 0 & 1 & 5 & 3 & 5 \\
\hline 3 & 3 & 4 & 4 & 0 & 1 & 0 & 0 & 3 & 3 & 4 & 6 \\
\hline 4 & 4 & 4 & 2 & 0 & 2 & 0 & 1 & 5 & 4 & 5 & 3 \\
\hline 5 & 2 & 2 & 1 & 0 & 0 & 1 & 0 & 8 & 3 & 4 & 7 \\
\hline $\begin{array}{l}\text { Total } \\
\text { frequency }\end{array}$ & 18 & 17 & 11 & 0 & 5 & 2 & 2 & 19 & 19 & 20 & 25 \\
\hline
\end{tabular}


S1 Drawing on background knowledge

S2 Drawing on context (relating the idiom to the wider familiar context like the whole paragraph)

S3 Translation (finding an L1 equivalent for the unfamiliar idiom or just translating it )

S4 Visualization (forming a mental image of the written idiom)

S5 Referring to the possible origin of the word in L1 or L2

S6 Wild guessing

S7 Requesting information (particularly the meaning of unfamiliar words)

S8 Paraphrasing and elaboration

S9 Focusing on key words

S10 Focusing on the literal meaning

S11 Drawing on the animated picture

Strategies 4 and 11 proved contexts dependent and mutually exclusive though both focused on images (whether mental or animated).Strategies5, 6 and 7 were relatively of low frequency in both groups(selected less than 10 times), while strategies number 2 and 11 were the most frequent ones in the text and animation groups respectively.

The questionnaire prepared based on the results of the think-aloud session (appendix A) was then administered to both groups. Table 3 and figure 1 show a cross-comparison between the frequencies of choosing each strategy in the questionnaire by the subjects of two groups. In table 3, strategy No.7 includes both focusing on the animated pictures, a strategy exclusively used by the subjects of the animation group, and visualization, the strategy specific to the text group. This was done for the ease of comparison as both strategies though different in nature shared the feature of focusing on images.

Table 3. The frequencies of different strategies used by the respondentsto the questionnaires

\begin{tabular}{llll}
\hline & & F. & F. \\
Strategies & Text & Animation \\
\hline S1 & Translation & 23 & 18 \\
S2 & Focusing on keywords & 81 & 74 \\
S3 & Paraphrasing and elaboration & 24 & 28 \\
S4 & Reference to the Literal meaning & 32 & 23 \\
S5 & Reference to context & 70 & 56 \\
S6 & Reference to the back ground knowledge & 45 & 40 \\
S7 & Focusing on the animated Picture/visualization & 37 & 80 \\
S8 & Combination of strategies & 41 & 55 \\
S9 & Other strategies & 11 & 6 \\
\hline
\end{tabular}

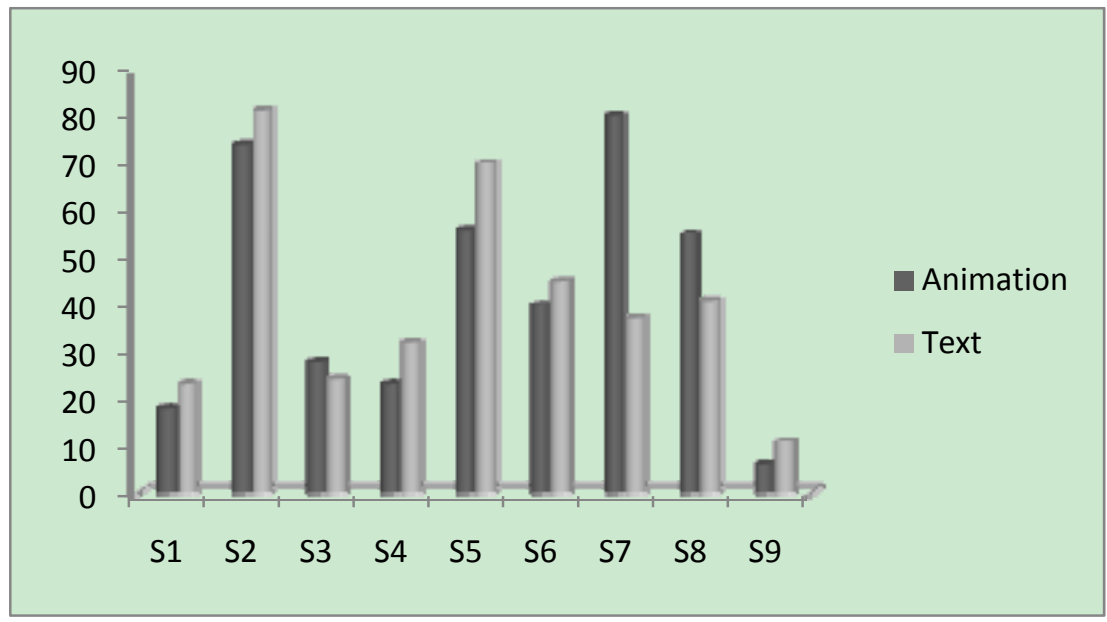

Figure 1. Paired frequencies of strategies based on the result of the questionnaire 
The results of the questionnaire roughly confirmed those of the think-aloud session though the two strategies of "paraphrasing and elaboration" and "reference to the literal meaning" displayed minor differences.

As table 3 shows, reference to animated pictures was of the highest frequency in the animation group while focusing on keywords was its counterpart in the text group. Translation to L1 takes the lowest position in the ranking of single strategies in both groups. Resorting to key words attains the highest rank if the preferences of both groups are taken into account.

Table 4 displays the order of the strategies referred to by the subjects of the two groups based on the descriptive data. The table arranged based on the frequencies, indicates differences in the order of strategies applied in the two groups.

Table 4. Strategies used by two groups in a descending order

\begin{tabular}{ll}
\hline Text group & Animation Group \\
\hline 1. Focusing on keywords & 1. focusing on the animated Picture \\
2 Reference to context & 2. Focusing on keywords \\
3. Reference to the back ground knowledge & 3. Reference to context \\
4. visualization & 4. Reference to the back ground knowledge \\
5. Reference to the Literal meaning & 5. Paraphrasing and elaboration \\
6. Paraphrasing and elaboration & 6. Reference to the Literal meaning \\
7. Translation & 7. Translation \\
\hline
\end{tabular}

The differences displayed in table 4 were based on descriptive statistics. To find out if such differences are statistically significant, intergroup and intra-group comparisons were made using chi-square tests. Table 5 displays the results of the inter-group comparison. As shown in the table, the two groups were significantly different in resorting to images, i.e. mental images vs. animated pictures. In other words, the subjects in the animation group regarded the images as their main source for reference (see figure 1) while this was not the same for the subjects in the text group. Comparison between the frequencies of other strategies also indicated differences though such differences didn't prove statistically significant.

Table 5. The chi-square results for intergroup comparison of paired strategies

\begin{tabular}{llll}
\hline Strategies & Chi-square & Asymp. Sig. & df \\
\hline 1. Translation & 0.610 & 0.435 & 1 \\
2. focusing on key words & 0.316 & 0.574 & 1 \\
3. Paraphrasing and elaboration & 0.490 & 0.484 & 1 \\
4. Reference to the literal meaning & 1.473 & 0.225 & 1 \\
5. Reference to the context & 1.556 & 0.212 & 1 \\
6. Reference to the background knowledge & 2.368 & 0.124 & 1 \\
7. focusing on the animated picture / Visualization & 16.407 & $0.000^{*}$ & 1 \\
8. Combined strategies & 2.042 & 0.153 & 1 \\
\hline
\end{tabular}

An intra-group comparison between the frequencies of different strategies shed further light on the context-dependent differences in processing idioms. Tables 6 and 7 show the pairs of strategies that proved significantly different in their frequency of use in the text and animation groups respectively.

Table 6. Pairs of strategies that proved significantly different in frequency of use by the text group

\begin{tabular}{llll}
\hline Intragroup strategy comparison (Text) & Chi-square & Asymp. Sig. & $\mathrm{df}$ \\
\hline 1. Translation \& background knowledge & 7.118 & .008 & 1 \\
2. key word \& back ground knowledge & 10.286 & 0.001 & 1 \\
3. background \& context & 5.435 & .020 & 1 \\
4. keyword \& paraphrase & 32.346 & 0.000 & 1 \\
5. Keyword \& visualization & 16.407 & 0.000 & 1 \\
6. Literal meaning \& context & 14.157 & 0.000 & 1 \\
7. Literal meaning \& keyword & 21.248 & 0.000 & 1 \\
8. Paraphrase \& background & 7.118 & 0.008 & 1 \\
9. Paraphrase \& Context & 23.753 & 0.000 & 1 \\
10. Translation \& Context & 23.753 & 0.000 & 1 \\
11. Translation \& keyword & 32.346 & 0.000 & 1 \\
\hline
\end{tabular}


Table 7. Pairs of strategies that proved significantly different in frequency of use by the animation group

\begin{tabular}{llll}
\hline Intragroup strategy comparison (Animation) & Chi-square & Asymp. Sig. & df \\
\hline 1. Animation \& background knowledge & 13.893 & 0.000 & 1 \\
2. key word\&paraphrase & 20.745 & 0.000 & 1 \\
3. Animation \& context & 5.321 & .021 & 1 \\
4. keyword \& Context & 2.492 & 0.014 & 1 \\
5. Keyword \& Background knowledge & 10.140 & 0.001 & 1 \\
6. Literal meaning \& Animation & 32.346 & 0.000 & 1 \\
7. Literal meaning \&Context & 13.785 & 0.000 & 1 \\
8. Literal meaning \&Keyword & 26.814 & 0.000 & 1 \\
9. Literal meaning \& Background knowledge & 4.587 & 0.032 & 1 \\
10. Paraphrase \& Animation & 25.771 & 0.000 & 1 \\
11. Paraphrase \& Context & 9.333 & 0.002 & 1 \\
12. Translation \& Animation & 40.091 & 0.000 & 1 \\
13. Translation \& Context & 19.514 & 0.000 & 1 \\
14. Translation \& Key word & 34.087 & 0.000 & 1 \\
15. Translation \& Background Knowledge & 8.345 & 0.004 & 1 \\
16. Translation \& Paraphrase & 2.174 & 0.040 & 1 \\
\hline
\end{tabular}

As shown in tables 6 and 7, while 11 pairs of strategies proved significantly different in the text group, the animation group indicated 16 cases of difference which in turn emphasizes the discrepancy between the processes involved.

\section{Discussion}

Identifying the strategies used in language learning has recently developed as a prominent research field. Empirical research has provided evidence that such strategies can be taught (Vance, 1999), which is a major reasons for language practitioners to focus on the strategies involved in learning different areas of language.

The present study was an attempt to shed further light on idiom processing by EFL learners. The think aloud session led to an inventory of strategies the subjects referred to in two different contexts. The two groups shared most of the strategies though they differed in the frequencies of using them. The frequency differences thus identified between the small samples were almost confirmed by the results obtained through administering the questionnaires to considerably larger samples. The descriptive data indicated differences both in frequencies and ranking of the strategies each group referred to in defining idioms. In other words, the primary results provided an inventory of the strategies the two groups referred to in defining idioms which in turn roughly answered the first two research questions. The descriptive results also indicated differences in the frequencies or types of strategies applied by the two groups, which is an answer to the third research question.However, going beyond the descriptive statistics further clarified the issue. Inferential statistics confirmed both inter-group and intra-group differences. The most significant difference in inter-group comparison was attributed to the two image based strategies, namely visualization and focusing on the animated pictures. Other similar strategies applied by the members of two groups were not significantly different in their frequencies. The intra-group comparison between the frequencies of pairs of strategies indicated even further differences. While the frequencies of the strategies used by the text group revealed 11 statistically different pairs, the animation group outnumbered the text group by indicating 16 cases of significant difference. So the results of the study while confirming the important role of context in language processing have some implications for language practitioners and material developers. Contextualizing language in general and idioms in specific can significantly influence the way language learners process the information. Therefore creating the appropriate context would prove productive in enhancing the language learning process.

The present study despite its attempt to control as many factors as possible, suffers from some shortcomings. Due to the limitations in the available materials, the idioms used in this study were not of a specific category following the classifications existing in the literature. The subjects of the study were both males and females and some strategies with lower frequencies were ignoredin the preparation of the questionnaire. In addition the present study just focused on the difference in the type and frequencies of the applied strategies with no regard to the correctness of the answers. Other studies are needed to focus on the effect of each context on enhancing the language learners' comprehension. In addition, as the present study only focused on single strategies, other studies are needed to investigate the combined strategies applied in comprehending idioms. 


\section{References}

Barkema, H. (1996). Idiomaticity and terminology: A multi-dimensional descriptivemodel. Studia Linguistica, 50(2), 125-160.

Boers, F., Eyckmans, J., \& Stengers, H. (2006). Means of motivating multiword units: rationale, mnemonic benefits and cognitive-style variables. In Foster-Cohen, S., Medved Krajnovic, M., \& Mihaljevic Djigunovic, J. (Eds.), EUROSLA Yearbook 6 (pp. 169-90). Amsterdam/Philadelphia: John Benjamins.

Boers, F., Lindstromberg, S., Littlemore, J., Stengers, H., \& Eyckmans, J. (2008). Variables in the mnemonic effectiveness of pictorial elucidation. In Boers, F., \& Lindstromberg, S. (Eds.), Cognitive linguistic approaches to teaching vocabulary and phraseology (pp. 189-216). Berlin/New York: Mouton de Gruyter.

Bobrow, S., \& Bell, S. (1973). On catching on to idiomatic expressions. Memory and Cognition, 1, 343-346. http://dx.doi.org/10.3758/BF03198118

Cacciari, C., \& Tabossi, P. (1988). The comprehension of idioms. Journal of Memory and Language, 27, 668-683. http://dx.doi.org/10.1016/0749-596X(88)90014-9

Collins COBUILD Dictionary of Idioms ( $2^{\text {nd }}$ ed.). (2002). Glasgow: Harper Collins.

Cooper, T. C. (1999). Processing of idioms by L2 learners of English.TESOL Quarterly, 33, 233-267. http://dx.doi.org/10.3758/BF03198118

Cowie, A. P. (1998). Introduction. In A. P. Cowie (Ed.), Phraseology: Theory, Analysis, and Applications (pp. 1-20). Oxford: Clarendon Press.

Ellis, R. (1997). Second Language Acquisition. Oxford: OUP.

Ericsson, K. A., \& Simon, H. A. (1993). Protocol analysis: Verbal reports as data (Rev. Ed.). Cambridge, MA: MIT Press.

Gibbs, R. W. (1980). Spilling the beans on understanding and memory for idioms. Memory \& Cognition, 8 , 449-456. http://dx.doi.org/10.3758/BF03213418

Gibbs, R. W., Jr. (1984). Literal meaning and psychological theory. Cognitive Science, 8, 275-304.

Gibbs, R. W., Jr. (1994). The poetics of mind, figurative thought, language, and understanding. Cambridge: Cambridge University Press.

Gibbs, R. (1999). Figurative language. In R. Wilson, \& F. Keil (Eds.), Themit encyclopedia of the cognitive sciences (pp. 314-315). Cambridge: MIT Press.

Gibbs, R. W., \& Nayak, N. P. (1989). Psycholinguistic studies on the synstrategy behavior of idioms. Cognitive Psychology, 21, 100-138.

Gibbs, R. W., Nayak, N. P., \& Cutting, C. (1989). How to kick the bucket and not decompose: Analyzability and idiom processing. Journal of Memory and Language, 28, 576-593. http://dx.doi.org/10.1016/0749-596X(89)90014-4

Gibbs, R. W., Nayak, N. P., Bolton, J. L., \& Keppel, M. E. (1989). Speakers' assumptions about the lexical flexibility of idioms. Memory \& Cognition, 17, 58-68. http://dx.doi.org/10.3758/BF03199557

Glaser, R. (1998). The stylistic potential of phraseological units in the light of genre analysis. In A. P. Cowie (Ed.), Phraseology: Theory, Analysis, and Applications (pp. 125-143). Oxford: Clarendon Press.

Glucksberg, S. (1993). Idiom meanings and allusional content. In C. Cacciari, \& P. Tabossi (Eds.), Idioms: Processing, structure, and interpretation (pp. 3-26). Hillsdale, NJ: Erlbaum.

Glucksberg, S. (2001). Understanding figurative language: From metaphors to idioms. Oxford: University Press.

Hernandez, A., Li, P., \& MacWhinney, B. (2005). The emergence of competing modules in bilingualism. Trends in Cognitive Science, 9, 220-225. http://dx.doi.org/10.1016/j.tics.2005.03.003

Irujo, S. (1986). Don't put your leg in your mouth: Transfer in the acquisition of idioms in a second language. TESOL Quarterly, 20, 287-304. http://dx.doi.org/10.2307/3586545

Kasper, G. (1998). Analysing verbal protocols. TESOL Quarterly, 32(2), 358-362. http://dx.doi.org/10.2307/3587591

Kathleen R. McKeown, \& Dragomir R. Radev. (2000). Collocations. In Robert Dale, HermannMoisl, \& Harold 
Somers (Eds.), A Handbook of Natural Language Processing. Marcel Dekker.

Kellerman, E. (1983). Now you see it, now you don't. In S. Gass, \& L. Selinker (Eds.), Language transfer in language learning (pp. 112-134). Rowley, MA: Newbury House.

Levorato, M. C., \& Cacciari, C. (1995). The effects of different tasks on the comprehension and production of idioms in children. Journal of Experimental Child Psychology, 60, 261-283. http://dx.doi.org/10.1006/jecp.1995.1041

Liu, D. (2008). Idioms. Descriptions, Comprehension, Acquisition, and Pedagogy. New York: Routledge.

Nippold, M. A., \& Rudzinski, M. (1993). Familiarity and transparancy in idiom explanation.A developmental study of children and adolescents. Journal of Speech and Hearing Research, 36, 728-737.

Nippold, M. A., \& Taylor, C. L. (1995). Idiom understanding in youth: Further examination of familiarity and transparency. Journal of Speech and Hearing Research, 38, 426-433.

Nunberg, G., Sag, I., \& Wasow, T. (1994). Idioms. Language, 70, 491-534.

Oxford Advanced Learner's Dictionary of Current English ( $8^{\text {th }}$ ed.). (2010). Oxford: Oxford University Press.

Hornby, A. S., Cowie, A. P., \& Lewis, J. W. (1974). Oxford advanced learner's dictionary of current English. London: Oxford University Press.

Paivio, A. (1986). Mental representations. Oxford: Oxford University Press.

Perez, E. (1981). Oral language competence improves reading skills of MexicanAmerican third graders. The Reading Teacher, 35, 24-27.

Qualls, C. D., O’Brien, R. M., Blood, G. W., \& Hammer, C. S. (2003). Contextual variation, familiarity, academic literacy, and rural adolescents' idiom knowledge. Language, Speech, and Hearing Services in Schools, 34, 69-79.

Schweigert, W. A. (1986). The comprehension of familiar and less familiar idioms. Journal of Psycholinguistic Research, 15, 33-45. http://dx.doi.org/10.1007/BF01067390

Swinney, D. A., \& Cutler, A. (1979). The access and processing of idiomatic expressions. Journal of Verbal Learning and Verbal Behavior, 18, 523-534. http://dx.doi.org/10.1016/S0022-5371(79)90284-6

Tabossi, T., \& Zardon, F. (1995). The activation of idiomatic meaning. In M. Everaert, E. van der Linden, A. Schenk, \& R. Schreuder (Eds.), Idioms: Structural and psychological perspectives (pp. 273-282). Hillsdale, NJ: Erlbaum.

Titone, D. A., \& Connine, C. M. (1994). Descriptive norms for 171 idiomatic expressions: familiarity, compositionality, predictability, and literality. Metaphor and Symbolic Activity, 9, 247-70. http://dx.doi.org/10.1207/s15327868ms0904_1

Titone, D. A., \& Connine, C. M. (1999). On the compositional and noncompositional nature of idiomatic expressions. Journal of Pragmatics, 31, 1655-1674. http://dx.doi.org/10.1016/S0378-2166(99)00008-9

Vance, S. J. (1999). Language learning strategies: Is there a best way to teach them? ERIC Document Reproduction Service No. ED438716.

\section{Appendix A}

\section{Dear Student:}

The present study aims at enhancing teaching and learning idioms by language learners. Please follow the instructions and fill in the questionnaire carefully.

Watch the animated cartoon and check the strategy (T1, T2, T3...) you follow to guess the meaning of each idiom paused at. The list of possible strategies is below the table. If the strategy you use is not in the list, choose "other". In case you apply more than one strategy, and you are sure all of them are equally important to lead you to its definition, check the last column on the right i.e. "combined". But if despite applying different strategies, just one is the main Strategy leading you to the definition of the idiom, only check that single major T(Strategy).

Familiarity: I'm already familiar with this idiom. (Choose the appropriate option to show how familiar it is.)

T1: Drawing on background knowledge

T2: Contextualizing: referring back to the previous sentences or parts of the story

T3: Translation: finding a Persian equivalent for the unfamiliar idiom or translating it to Farsi 
T4: Animated cartoon elucidation: Focusing on that specific part of the cartoon to define the idiom

T5: Paraphrasing and elaboration: simplifying and rephrasing the idiom to make the meaning clear

T6: Focusing on (a) key word(s): choosing specific words as the main or key words of the idiom to guess its meaning

T7: Focusing on the literal meaning: guessing the meaning of idiom by focusing on its literal (non-idiomatic) meaning

T8: Combination of strategies (read the instructions above.)

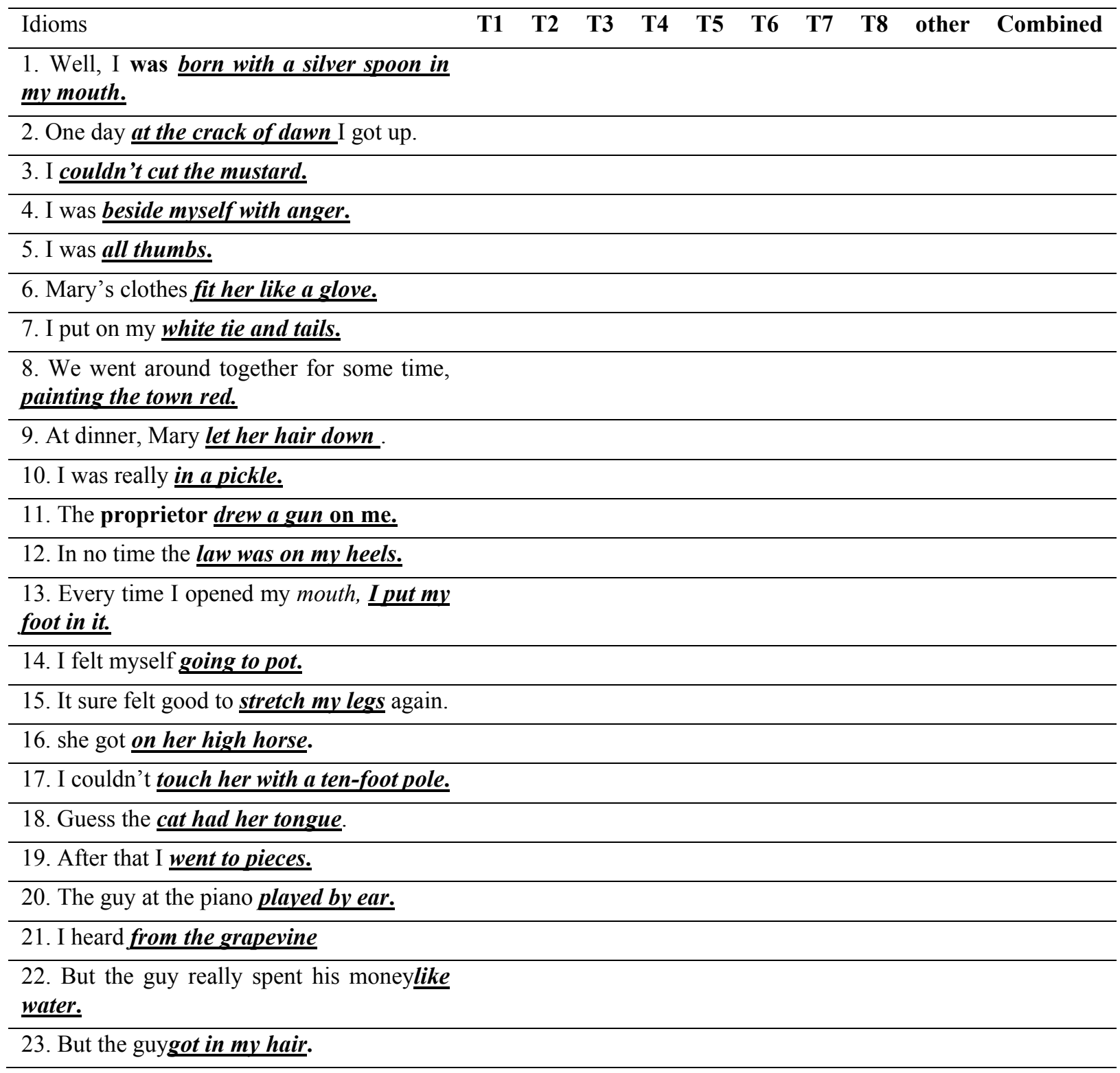

Thank you for your attention. 